\title{
Asian Consensus Guidelines for the Diagnosis and Management of Gastrointestinal Stromal Tumor
}

\author{
Dong-Hoe Koo, MD, $\mathrm{PhD}^{1,2}$ \\ Min-Hee Ryu, MD, PhD',3 \\ Kyoung-Mee Kim, MD, PhD2,4 \\ Han-Kwang Yang, MD, PhD2,5 \\ Akira Sawaki, MD, $P h D^{6,7}$ \\ Seiichi Hirota, MD, PhD 7,8 \\ Jie Zheng, $M D, P h D^{9,10}$ \\ Bo Zhang, MD, PhD 10,11 \\ Chin-Yuan Tzen, MD, PhD'12 \\ Chun-Nan Yeh, MD ${ }^{13}$ \\ Toshirou Nishida, MD, PhD 7,14 \\ Lin Shen, MD, PhD ${ }^{10,15}$ \\ Li-Tzong Chen, MD, PhD 16,17 \\ Yoon-Koo Kang, MD, PhD 2,3
}

*A list author's affiliations appears at the end of the paper.

Correspondence: Yoon-Koo Kang

Department of Oncology, Asan Medical Center,

University of Ulsan College of Medicine,

88 Olympic-ro 43-gil, Songpa-gu,

Seoul 05505, Korea

Tel: 82-2-3010-3210 Fax: 82-2-3010-8772

E-mail: ykkang@amc.seoul.kr

Co-Correspondence: Toshirou Nishida

Department of Surgery, National Cancer Center

Hospital East, 6-5-1 Kashiwanoha, Kashiwa,

Chiba 277-8577, Japan

Tel: 81-4-7133-1111- Fax: 81-4-7134-8676

E-mail: tnishida@east.ncc.go.jp

Co-Correspondence: Lin Shen

Department of Gastrointestinal Oncology

Peking University Cancer Hospital and Institute,

Fu-Cheng Road 52, Hai-Dian District,

Beiiing 100142, China

Tel: 86-10-8819-6561 Fax: 86-10-8819-6561

E-mail: lin100@medmail.com.cn

Co-Correspondence: Li-Tzong Chen

National Institute of Cancer Research, National Health

Research Institutes, and Department of Internal

Medicine, National Cheng Kung University Hospital,

National Cheng Kung University, No.367,

Sheng-Li Road, Tainan 70456, Taiwan

Tel: 886-6-700-0123 Fax: 886-6-208-3427

E-mail: leochen@nhri.org.tw

Received April 22, 2016

Accepted June 3, 2016

Published Online June 24, 2016

${ }^{*}$ Dong-Hoe Koo and Min-Hee Ryu contributed equally to this work.
Gastrointestinal stromal tumors (GISTs) are the most common mesenchymal tumors originating in the gastrointestinal tract. With the introduction of molecular-targeted therapy for GISTs which has yielded remarkable outcomes, these tumors have become a model of multidisciplinary oncological treatment. Although Western clinical guidelines are available for GISTs, such as those published by the National Comprehensive Cancer Network (NCCN) and the European Society of Medical Oncology (ESMO), the clinical situations in Asian countries are different from those in Western countries in terms of diagnostic methods, surgical approach, and availability of new targeted agents. Accordingly, we have reviewed current versions of several GIST guidelines published by Asian countries (Japan, Korea, China, and Taiwan) and the NCCN and ESMO and discussed the areas of dissensus. We here present the first version of the Asian GIST consensus guidelines that were prepared through a series of meetings involving multidisciplinary experts in the four countries. These guidelines provide an optimal approach to the diagnosis and management of GIST patients in Asian countries.

\section{Key words}

Guideline, Gastrointestinal stromal tumors, Imatinib mesylate, Sunitinib, Regorafenib 


\section{Introduction}

Gastrointestinal stromal tumors (GISTs) are the most common mesenchymal tumors originating in the gastrointestinal tract. They are generally positive for CD117 (c-Kit), and are primarily caused by activating mutations in KIT or PDGFRA with a characteristic morphology [1]. The identification of a signal transduction pathway associated with the development of GISTs facilitated advances in the understanding of this tumor. Indeed, molecular-targeted therapy with imatinib (Glivec, Novartis) has yielded remarkable outcomes. GISTs have become a model of multidisciplinary oncological treatment, and an interdisciplinary approach is required to provide optimal care for GIST patients. Surgical and medical oncology, pathology, and gastroenterology are the essential fields of the multidisciplinary treatment of GIST.

Although Western clinical guidelines for the treatment and management of GISTs are available, such as those published by the National Comprehensive Cancer Network (NCCN) and the European Society of Medical Oncology (ESMO) [2,3], it is unclear whether these guidelines are appropriate for the clinical situation in Asian countries. Despite the limited Asian data on GISTs, several studies have suggested that some aspects of Western guidelines are not applicable to Asian GIST patients and that clinical practice patterns in Asia differ from those of Western countries [4-7]. Therefore, consensus meetings were held involving multidisciplinary experts including surgeons, pathologists, and medical oncologists from Korea, Japan, China, and Taiwan since April 2014. These Asian GIST experts thoroughly reviewed the relevant Asian literature and shared their experience and opinion to reach a consensus on topics related to the pathologic diagnosis and surgical and medical treatment of Asian GIST patients.

The present consensus guidelines aimed to provide an optimal approach to the diagnosis and management of GIST patients in Asian countries.

\section{Pathologic Diagnosis of GISTs}

Although GISTs can arise in any portion of the gastrointestinal tract, they usually occur in the stomach $(60 \%)$ or small intestine (30\%). GISTs rarely occur in extra-gastrointestinal locations such as the omentum, mesentery, pelvis, and retroperitoneum [8]. GISTs range in size from less than $1 \mathrm{~cm}$, typically discovered incidentally during tests for other diseases, to very large lesions measuring up to $35 \mathrm{~cm}$ (median, $5 \mathrm{~cm}$ ) [9]. Regardless of their size, GISTs have typ-

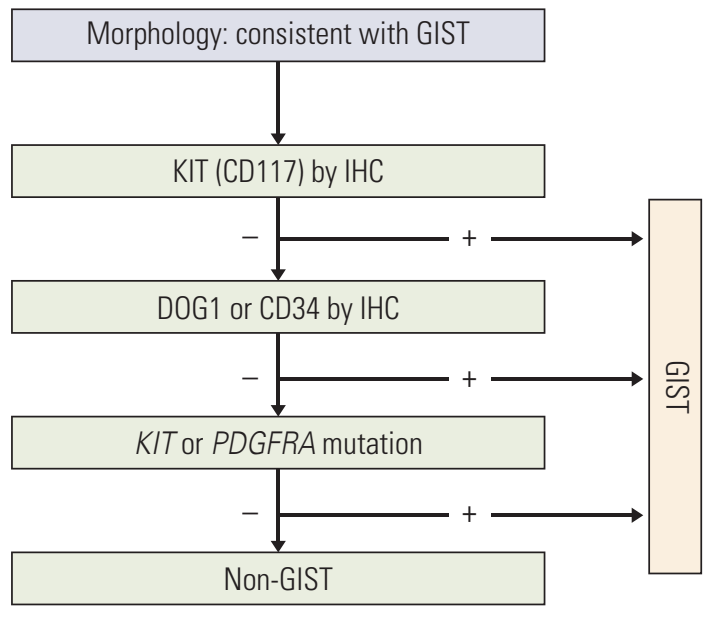

Fig. 1. Algorithm for the diagnosis of gastrointestinal stromal tumor (GIST). IHC, immunohistochemistry.

ical morphologic features and immunoreactivity for c-Kit (CD117), and may contain oncogenic mutation in the KIT (80\%-85\%) or platelet-derived growth factor receptor alpha (PDGFRA; 5\%-7\%) genes [10].

\section{Morphology and immunohistochemical staining}

Macroscopically, GISTs are typically a well-circumscribed fleshy, pink or tan-white mass. Large tumors frequently present with hemorrhage, necrosis, and cystic degeneration. GISTs can be divided into three different histologic subgroups; spindle cell GISTs (70\%) which consist of cells with pale eosinophilic fibrillary cytoplasm, ovoid uniform nuclei, and ill-defined cell borders; epithelioid GISTs (20\%) which consist of rounded cells with eosinophilic to clear cytoplasm arranged in sheets and nests; and the mixed type (10\%) with spindle and epithelioid cells.

The pathological diagnosis of GIST is mainly based on the histological profile of the tumor (recommended to be obtained via surgical resection rather than preoperative biopsy), but immunohistochemical (IHC) staining is needed to confirm the diagnosis [11]. The most important IHC staining method for the diagnosis of GISTs is c-Kit (CD117) but staining with several other antibodies may also be helpful for primary and differential diagnosis. Approximately $94 \%-98 \%$ of GISTs are positive for the c-Kit protein, which is rarely expressed in other abdominal tumors. In addition, CD34 is positive in $60 \%-80 \%$ of all GISTs. A recently developed antibody against DOG1 (discovered on GIST) was reported to be positive in $85 \%-95 \%$ of c-Kit-positive GISTs and in 30\%-36\% of c-Kit-negative GISTs [12]. DOG1 expression is not different between KIT/PDGFRA mutant GIST and wild-type GIST. 
Therefore, DOG1 immunostaining may be helpful for tumors which cannot be diagnosed as GIST based on c-Kit immunohistochemistry.

The algorithm for GIST diagnosis drawn by consensus is provided in Fig. 1. First, the morphology should be consistent with that of a GIST. Second, if IHC staining for c-Kit is positive, then the tumor can be diagnosed as GIST. If the c-Kit stain is negative but the DOG1 IHC stain is positive, the tumor can be diagnosed as GIST. Gastrointestinal mesenchymal tumors that harbor a KIT or PDGFRA mutation can also be diagnosed as GIST, even though they are negative for both c-Kit and DOG1. For gastrointestinal mesenchymal tumors not diagnosed as GIST according to the above criteria, other IHC markers such as CD34, smooth muscle actin (SMA), S-100, desmin, and protein kinase C (PKC)-theta can be used for differential diagnosis.

c-Kit negative GISTs account for approximately $5 \%$ of all cases [13]. Among the c-Kit-negative GISTs, 40\%-45\% are positive for CD34, 75\% are positive for PKC-theta, and 30\%$40 \%$ are positive for SMA $[13,14]$. S-100 and desmin staining are usually recommended to exclude neural tumors and smooth muscle tumors, respectively.

\section{Mutational analysis}

KIT mutations ( $80 \%$ of primary GISTs) are most common in exon $11(65 \%)$, followed by exon $9(10 \%)$, and are rarely found in exons 13 and 17. PDGFRA mutations (10\% of primary GISTs) are common in tumors of the stomach and are associated with epithelioid features and indolent behavior [1]. The most common mutation of PDGFRA is exon 18 $\mathrm{D} 842 \mathrm{~V}$ and is associated with resistance to imatinib. At present, mutation analysis is not required for the diagnosis of GISTs when the tumors have the typical histology and c-Kit and/or DOG-1 positivity. However, mutation analysis should be considered when diagnosing immunohistochemically c-Kit negative GISTs. In addition, mutation analysis can be recommended to predict treatment response to imatinib [15-17], especially to exclude imatinib-resistant GIST (D842V) in neoadjuvant or adjuvant setting or to determine the initial dose of imatinib for GISTs with a KIT exon 9 mutation [10]. Mutational analysis for KIT exons 9, 11, 13, and 17 and PDGFRA exons 12, 14, and 18 can be performed using both formalin-fixed, paraffin-embedded tissue and fresh-frozen tissue.

Although most GISTs are driven by KIT- or PDGFRA-activating mutations, a small subset $(10 \%)$ without KIT or PDGFRA mutations exist and are referred to as wild-type GISTs which may harbor mutations in succinate dehydrogenase $(S D H)$ complex including SDHA, SDHB, SDHC, or SDHD, BRAF, KRAS, or NRAS [1,18,19]. SDH-deficient GISTs in particular, include pediatric cases and those associated with Carney triad syndrome (gastric epithelioid GIST, pulmonary chondroma, and extra-adrenal paraganglioma in young females) or Carney-Stratakis syndrome [20,21]. SDHdeficient GISTs, which most commonly occur in the stomach, have histologic features that are otherwise rare in gastric GISTs, such as epithelial hypercellular features, plexiform growth patterns in the muscularis propria, and lymphovascular invasion and lymph node metastases [21].

\section{Risk classification in localized GISTs}

Risk assessment of recurrence after curative surgery for localized GISTs provides the basis for optimal adjuvant treatment. The two most important prognostic factors are mitotic index and tumor size as suggested by the National Institutes of Health (NIH) consensus criteria published in 2002 [11]. The Armed Forces Institute of Pathology (AFIP) criteria added tumor location to size and mitotic count [22].

Table 1. Modified NIH classification system proposed by Joensuu [23]

\begin{tabular}{|c|c|c|c|}
\hline Risk category & Tumor size $(\mathrm{cm})$ & Mitotic index (/50 HPFs) & Primary tumor site \\
\hline Very low risk & $\leq 2.0$ & $\leq 5$ & Any \\
\hline Low risk & 2.1-5.0 & $\leq 5$ & Any \\
\hline \multirow[t]{2}{*}{ Intermediate risk } & $\leq 5.0$ & $6-10$ & Gastric \\
\hline & $5.1-10.0$ & $\leq 5$ & Gastric \\
\hline \multirow[t]{6}{*}{ High risk } & Any & Any & Tumor rupture \\
\hline & $>10.0$ & Any & Any \\
\hline & Any & $>10$ & Any \\
\hline & $>5.0$ & $>5$ & Any \\
\hline & $\leq 5.0$ & $>5$ & Non-gastric \\
\hline & $5.1-10.0$ & $\leq 5$ & Non-gastric \\
\hline
\end{tabular}

Adopted from Joensuu H. Hum Pathol. 2008;39:1411-9, with permission of Elsevier [23]. NIH, National Institutes of Health; HPFs, high-power fields. 
The modified NIH classification suggested by Joensuu [23] combined the advantages of the NIH (tumor size and mitosis) and AFIP (tumor location) criteria along with the additional factor of rupture (Table 1) [24]. Moreover, a novel prognostic contour map was generated through the pooled data of GIST patients who received no adjuvant therapy [25]. The contour map incorporated the mitotic index and tumor size as continuous non-linear variables while tumor rupture and primary site were included as categorical variables for risk assessment.

Given these differences in the various risk classifications, it remains unclear which classification is the best for selecting candidates for adjuvant therapy. Our expert panel recommends using the modified NIH criteria to guide the selection of patients who may require adjuvant therapy, on the basis of the four risk factors including size, mitosis, primary site, and rupture. In addition, a recent Japanese report indicated that the modified NIH criteria was the most sensitive for detecting patients who are likely to have recurrence [26].

\section{Pathologic reporting for GISTs}

The pathology report for GISTs should include the following: (1) tumor location and size and mitotic index (per 50 high-power fields); (2) resection margin status; (3) presence or absence of metastases or rupture during the operation; and (4) IHC staining results that include CD117 (c-Kit) with or without CD34/DOG-1. It must be considered that the actual size of a high-power field may differ between microscopes when obtaining mitotic counts. Hence, the panels recommend that mitotic counts are expressed as per $5 \mathrm{~mm}^{2}$ fields in near future. In addition, a pathology report may include other histological nature of the tumor, including the degree of cellularity, presence of necrosis or cystic changes, and any invasion into the mucosa or adjacent structures.

In terms of pathologic reporting, there were some different circumstances in Asia that do not exactly match the Western guidelines such as the NCCN and ESMO guidelines. First, genotyping for KIT or PDGFRA is strongly recommended in these guidelines, but genotyping is not widely used and standardized in many centers in Asia. Therefore, we recommend that the tumor tissue should be referred to centers where genotyping facility is available, especially, in specific situations such as to diagnose immunohistochemically negative GISTs or to predict treatment response to imatinib. Second, we recommend using the modified NIH criteria among the various risk classifications, especially to select patients who may require adjuvant imatinib therapy for 36 months. Third, we proposed to report at least four items in pathology report because, in some cases, insufficient information can be provided by a pathologist who is not well-experienced with GISTs.

\section{Surgical Treatment of GISTs}

\section{Preoperative evaluation and biopsy}

Surgical resection is the current standard of care for localized GISTs. The initial diagnosis is generally suggested by endoscopy, endoscopic ultrasound (EUS), or computed tomography (CT) of the abdomen, which should be confirmed histopathologically after surgical resection of the tumor. Preoperative histological diagnosis is feasible but may sometimes be difficult to interpret [27]. Imaging studies used to detect metastasis include triphasic CT of the abdomen and pelvis and / or magnetic resonance imaging, as necessary [28]. Chest CT is rarely needed for initial evaluation of GISTs because extra-abdominal metastasis at initial presentation is extremely rare. Positron emission tomography (PET) may be performed when evidence of metastasis is equivocal or for the purpose of conducting clinical trials [27].

Biopsy is absolutely necessary when considering initial imatinib therapy in terms of neoadjuvant treatment. Preoperative biopsy should be performed, with effort to avoid tumor spillage or metastases. In this regard, EUS-guided biopsy is preferred to percutaneous biopsy [29]. If the tumor is strongly suggestive of GIST and is considered resectable, preoperative biopsy can be omitted [30]. In metastatic diseases, however, percutaneous image-guided biopsy may be allowed. When the histology shows unusual or complex features, the diagnosis should be reviewed by an expert to discriminate GISTs from other sarcomas. Mutation analysis is helpful and is recommended if possible.

\section{Indications for surgery}

When a tumor is suggestive of GIST, it should be considered for curative surgical resection due to the high malignant potential $[4-7,31]$. Surgery is the treatment of choice for patients with localized or potentially resectable GISTs. In gastric GISTs, surgical resection is strongly recommended when (1) tumors are $2 \mathrm{~cm}$ or larger in size, or (2) they are growing or have signs of malignancy, such as irregular margin, ulceration, bleeding, cystic change, necrosis, or heterogeneous echogenicity in endoscopy and / or EUS [27]. Smaller tumors $(<2 \mathrm{~cm})$ with no signs of malignancy may be managed with active surveillance. However, a small tumor size does not exclude the potential for malignancy in GIST. Therefore, patients should be informed about the possibility of malignancy, even if the tumor is small. For non-gastric GISTs, on the other hand, surgical resection is recommended, regardless of tumor size or morphology. 


\section{Surgical margins, lymph node dissection, and laparo- scopic surgery}

For complete resection, pseudocapsule should not be damaged during manipulation of the tumor and macroscopically negative margin as well as adequate safety margin should be spared. In cases with tumor infiltration into the surrounding organs, a complete en bloc resection with negative margins should be performed, regardless of tumor size $[29,31]$. When adjacent organs adhere to the tumor, complete en bloc resection should also be performed to avoid tumor rupture or intra-abdominal spillage [29]. En bloc resection is also recommended for omental and mesenteric GISTs. In gastric GISTs, subtotal or total gastrectomy may be performed based on the tumor size and location. In many cases, wedge resection of gastric GISTs and segmental resection of small bowel GISTs are sufficient; however, simple enucleation or endoscopic treatment is not recommended even if the GIST is small.

When the macroscopic surgical margin is positive ( $\mathrm{R} 2$ disease), reoperation is recommended when possible. In cases of positive microscopic resection margin (R1), postoperative imatinib therapy is recommended when the malignant potential-based on the size, mitotic index, and primary site of the GIST-is high. If the risk of recurrence is low, routine surveillance with no additional therapy is recommended. A retrospective analysis has suggested that margin status might not be a significant prognostic factor for recurrencefree survival in the era of targeted therapy [32].

Since GISTs rarely spread to local or regional lymph nodes, lymph node dissection is usually not necessary, but it should be considered if metastasis is suspected, such as when enlarged lymph nodes are noted.

Although the evidence to date is mainly limited to gastric GISTs, laparoscopic surgery can be performed for small GISTs in favorable locations, such as those located in the greater curvature or anterior wall of the stomach, jejunum, or ileum [33]. Tumor grasping and tumor rupture should be avoided; however, use of the stapling technique and extraction bags is recommended. Compared with open surgery, laparoscopic surgery has similar outcomes for GIST patients in terms of oncologic prognosis with several advantages, such as less pain, less invasiveness, early recovery, and better cosmetic results [34].

\section{Postoperative follow-up and surveillance}

For GIST patients in high- or intermediate-risk groups, postoperative surveillance is recommended with abdominal and pelvic CT scan every 3 to 4 months for the first 3 years after surgery, every 6 months until 5 years after surgery, and an annual assessment thereafter $[27,29]$. For patients in the low- or very low-risk groups, follow-up CT every 6 months is recommended for 5 years after surgery. Ultrasonography may replace CT once each year $[27,29]$. The role of PET in postoperative surveillance has not been established. Before the introduction of adjuvant therapy, most recurrences of GIST occurred in the liver and peritoneum within 2 years of surgery [35]. In the era of adjuvant imatinib therapy, a Western study reported that the risk of recurrence was also high within the first 1-2 years after adjuvant therapy [36]. Hence, further investigations are needed to establish an optimized surveillance schedule with CT in Asian countries.

\section{Surgery during tyrosine kinase inhibitor therapy for advanced GISTs}

Medical treatment using tyrosine kinase inhibitor (TKI) alone rarely achieves a complete response in advanced GISTs, as resistant tumor clones develop continuously over time after the initiation of imatinib treatment [37]. In particular, macroscopic residual lesions would contain many viable tumor cells; therefore, surgical removal of these residual lesions would prevent or delay the emergence of resistant clones. The survival benefit of adding surgical resection to imatinib at maximal response, including partial response (PR) and stable disease (SD), has been suggested in several retrospective studies, in which some patients maintained their disease status free from progression for a long while after surgery [38,39]. A prospective randomized comparative trial was carried out in China, although it was terminated early due to poor accrual $(n=41)$, it reported that 2-year progression-free survival (PFS) was $88.4 \%$ in patients who received surgery for residual disease with imatinib and $57.7 \%$ in patients who received imatinib alone $(\mathrm{p}=0.089)$. Median overall survival (OS) was not reached in the surgery arm of that study but it was 49 months in the imatinib alone $\operatorname{arm}(\mathrm{p}=0.024)$ [40]. Recently, Park et al. [41] conducted a retrospective comparative study in 134 patients who had SD for more than 6 months after responding to imatinib and reported that surgical resection of residual lesions was beneficial with longer PFS and OS compared with patients treated with imatinib alone. There is no definitive evidence from large phase III trials; however, the potential benefit of post-imatinib resection in preventing or delaying the development of imatinib-resistance has been strongly suggested and it is unlikely to have a large scale prospective randomized trial, this issue should be discussed with a multidisciplinary team or with experts in referral hospitals $[38,39,41]$. There is no evidence to recommend surgery during sunitinib or regorafenib.

Post-imatinib resection can be recommended when a GIST patient shows (1) a PR or SD after an adequate duration (usually 4 to 12 months) of imatinib therapy and residual lesions 
are completely resectable or (2) limited (or focal) progression of disease confined locally such as "nodule within the mass" [38]. In addition to surgical resection, hepatic or peritoneal metastases of GIST can be managed with local modalities, such as radiofrequency ablation or chemoembolization. TKI therapy must continue even after complete resection of the residual tumor.

In contrast to resection after imatinib therapy, initial debulking surgery before imatinib therapy is not beneficial [42]. In metastatic GISTs, surgery should be avoided as a primary approach and imatinib therapy is the standard primary treatment.

In this section, we emphasized aggressive surgery such as complete en bloc resection of resectable tumors including omental and mesenteric GISTs, which may seem to contradict with the Western guidelines in which points out to avoid multivisceral resection. However, the emphasis on aggressive surgery is only limited in curative setting of primary surgery, we also recommend to avoid mutilating surgery and consider preoperative imatinib when appropriate. In metastatic GISTs, even though all tumors seem completely resectable, we strongly recommend multidisciplinary approach for initial imatinib therapy because there is no evidence of survival benefit by initial debulking surgery prior to imatinib therapy.

\section{Medical Treatment of GISTs}

\section{Adjuvant treatment}

The purpose of adjuvant therapy is to reduce or delay the growth of microscopic tumors after complete resection of a GIST. A randomized, double-blinded, placebo-controlled phase III study (ACOSOG Z9001) showed that adjuvant imatinib therapy for 1 year could significantly prolong the recurrence-free survival of patients with primary GIST after curative resection [43]. A non-randomized, prospective phase II study also reported that adjuvant imatinib for 36 months decreased the risk of recurrence and death compared to observation group in Chinese GIST patients with intermediate or high risk [44]. Furthermore, a large, randomized, phase III trial (SSG XVIII/AIO) reported that adjuvant imatinib for 36 months reduced the risk of recurrence and improved OS compared with adjuvant imatinib for 12 months in GIST patients with high risk of recurrence after surgery [45]. Therefore, adjuvant imatinib is currently indicated in patients with high-risk GIST and is recommended for 3 years. For intermediate-risk patients, evidence is not sufficient to make such a conclusion. The modified NIH cri- teria are recommended for selecting adjuvant imatinib candidates, because the SSG XVIII/ AIO trial used the criteria for estimating the risk of GIST recurrence [45].

In addition to risk assessment, we recommend genotyping to identify the PDGFRA exon 18 D842V mutation, at least for primary gastric GISTs, if this method is available. Adjuvant imatinib is not recommended for cases with the D842V mutation because these tumors do not respond to imatinib. There is currently no consensus on whether wild-type GISTs should be treated with adjuvant therapy because of their lower sensitivity to imatinib and an often more indolent clinical course, especially in the case of syndromic GISTs such as neurofibromatosis type 1-related GISTs, Carney triad or Carney-Stratakis syndrome $[20,46]$.

\section{Neoadjuvant treatment}

Neoadjuvant imatinib therapy should be considered (1) for localized (not metastatic) GISTs when R0 resection is not feasible or (2) for organ function preservation, including the rectum, esophagus, or duodenum, or to avoid total gastrectomy $[47,48]$. Early assessment of tumor response by CT and/or PET after the first month of imatinib treatment is recommended to avoid delayed detection of rapid tumor progression due to primary imatinib resistance [49]. Surgery is recommended after achieving the best response or sufficient shrinkage of the tumor [27]. Thus, the duration of preoperative imatinib is usually 4-6 to 12 months. In the absence of significant imatinib-related adverse events, withholding imatinib before surgery is not necessary because imatinib does not have known negative impact on wound healing and safety of surgery.

After surgical resection of a GIST with pre-operative imatinib, the decision to commence adjuvant imatinib should be based on the pre-neoadjuvant tumor rather than post-imatinib tumor status. However, it can be difficult to classify risk because the pre-neoadjuvant biopsy tissues are often inadequate for determining the mitotic index. Adjuvant imatinib should be started as soon as the patient is allowed to have oral intake. The duration of adjuvant imatinib should be determined by the duration of pre-operative therapy. The total duration of imatinib therapy before and after surgery is at least 3 years for high risk GIST. If genotyping is available, tumors exhibiting PDGFRA exon 18 D842V mutation should be excluded from pre- and post-operative adjuvant imatinib therapy. However, if genotyping is not feasible and there is a possibility of the presence of $\mathrm{D} 842 \mathrm{~V}$ mutation, particularly for primary gastric GIST, neoadjuvant imatinib should be administered carefully and the tumor response should be evaluated earlier or as soon as possible. 


\section{First-line imatinib therapy for advanced GISTs}

Once an advanced GIST (metastatic, unresectable, or recurrent) is diagnosed, imatinib should be immediately prescribed; regardless of presence of symptoms and even if the tumor is completely resected grossly and histologically [35]. Cytoreductive surgery prior to imatinib therapy is not recommended [42]. The optimal initial dose of imatinib is 400 $\mathrm{mg}$ per day. A higher dose ( $800 \mathrm{mg}$ per day) of imatinib is now recommended as the initial treatment in Western countries for patients with KIT exon 9 mutant GISTs [15]. However, there was no large prospective study to evaluate the clinical benefit of higher-dose imatinib treatment in Asian patients with different genotypes [50,51]. A recent Korean large-scale retrospective analysis has suggested that patients with the KIT exon 9 mutation would also benefit from treatment with a higher dose of imatinib [17]. Our expert panel agreed with the recommendations of Western guidelines and suggests that a higher dose may also be beneficial in Asian patients with KIT exon 9 mutation. However, prospective trials are needed to prove the feasibility and efficacy of higherdose imatinib in Asian populations [52].

The most common adverse effects of imatinib are fluid retention, diarrhea, abdominal pain, nausea, poor appetite, fatigue, muscle cramps, and skin rash. These adverse effects usually improve and can be tolerable with prolonged imatinib therapy [53]. Discontinuation of imatinib treatment in patients with initial favorable tumor response generally leads to rapid disease progression $[54,55]$. In such cases, many patients may respond to the reintroduction of imatinib, but the tumor response may be smaller than what was achieved prior to treatment interruption [56]. Therefore, the expert meeting recommended giving imatinib treatment continuously until disease progression, intolerable adverse events occur, or the patients' refusal.

\section{Evaluation of the tumor response}

To date, CT has been the most useful diagnostic tool for evaluating the GIST response to TKI treatment. We recommend contrast-enhanced, dynamic or triphasic CT scanning with arterial and portal venous phases [27]. The interval for radiological tumor response evaluation may vary according to the clinical situation, but is usually performed every 3 to 6 months after the documentation of initial response. Fluorodeoxyglucose (FDG) PET is highly sensitive for evaluating early tumor response, but given its cost and lack of availability, there is no consensus on its inclusion in basic imaging tests [57].

The tumor response assessment should not solely rely on the change of tumor size because tumors can be enlarged with cystic changes secondary to intratumor hemorrhage or myxoid degeneration $[27,58]$. In addition, new hypoattenuating lesions resulting from reduced vascularity and hyaline degeneration can be observed following imatinib treatment. Particularly for cases of liver metastasis, new small lesions with hypodensity and clear margins may be observed in portal venous phase of CT scan resulting from necrosis of preexisting, small iso-dense tumors. Hence, caution is required when applying the Response Evaluation Criteria in Solid Tumors (RECIST) or World Health Organization (WHO) criteria for evaluating the response of GIST to TKI treatment. The development of new criteria, preferably taking both tumor size and density into account, is therefore warranted. An improvement in symptoms, reduction in the degree of the CT attenuation coefficient (Hounsfield units), or change in the maximum standardized uptake value (SUVmax) upon PET may also be used to determine the tumor response $[57,58]$.

Recurrence or progression includes the appearance of a new lesion at the surgery site, the development of new metastases, or an enlargement of the preexisting, hypervascular tumor. A new intratumoral nodule or increased solid tissue density within a previously responding, hypodense GIST is also considered as radiological evidence of tumor recurrence or progression. This pattern of progression is not defined in RECIST or WHO criteria, but all the experts agreed that such radiological changes are early signs of tumor progression. Therefore, the interior and cystic walls of previously responding hypodense tumors should be carefully examined [28,37]. The evaluation criteria require standardization and further validations in large-scale prospective studies are needed.

\section{Failure pattern of first-line treatment}

Tumor resistance to imatinib is classified as either primary or secondary. Primary resistance is defined as progression within the first 6 months of imatinib therapy, with most cases progressing multifocally [27]. Secondary resistance is defined as progression beyond 6 months after the initiation of imatinib therapy. In progressing lesions of imatinib resistant GISTs, secondary mutations in KIT including exon 13, 14, or 17 are commonly found, and represent a major factor of secondary resistance to imatinib [59].

There are two types of progression, as described below [27].

\section{1) Limited (or focal) progression}

Focal progression occurs when one lesion or a limited number of lesions exhibit intratumoral nodules or increased size with increased FDG uptake on PET whilst the other lesions remain relatively well controlled. Treatment of focal 
progression requires multidisciplinary approach. Local therapy, such as radiofrequency ablation, chemoembolization, or resection of localized progression of liver metastasis, or peritoneal tumor can be considered for the control of focally progressing imatinib-resistant lesions, while imatinib at standard dose is continued to control the remaining imatinibresponding tumors. If those lesions are not removable, escalation of the dose of imatinib or a switch to sunitinib should be considered. Although there was no prospective study to evaluate the efficacy of local treatment for focal progression in GIST, retrospective analyses have suggested that some cases with focal progression may benefit from local treatment $[38,60]$. On the other hand, there was a study showing the potential risk of rapid disease progression within a short period of time after local treatment [39].

\section{2) General (or multifocal) progression}

General progression is characterized by the simultaneous progression of most lesions. The efficacy of local treatment of general progression in GIST is extremely limited and mostly negative [38], therefore, it is not recommended, except for the purpose of symptomatic palliation. Administration of imatinib at increased doses or switch to a secondline drug such as sunitinib is indicated.

\section{Further treatment after failure of first-line imatinib}

\section{1) Higher dose of imatinib}

Approximately $30 \%$ to $40 \%$ of patients who had disease progression during imatinib treatment at standard dose of $400 \mathrm{mg}$ per day may benefit from an increased dose of imatinib to $600-800 \mathrm{mg}$ per day for a limited period [61]. With a daily dose of $800 \mathrm{mg}$, no increase in adverse events associated with imatinib has been found, other than malaise and anemia. If intolerable side effects occur, the dose may be reduced to $600 \mathrm{mg}$ per day [61]. When severe adverse events are anticipated with a direct dose escalation to $800 \mathrm{mg}$ per day, imatinib can be increased in stages, to $600 \mathrm{mg}$ per day, then to $800 \mathrm{mg}$ per day. The median PFS is approximately 3-5 months and the 12-month PFS rate ranges 18\% to 30\% with dose escalation in imatinib-resistant GISTs [61-63].

\section{2) Second-line sunitinib}

Sunitinib is a TKI that inhibits the vascular endothelial growth factor receptor as well as the KIT and PDGFRA. As a result, this drug inhibits tumor growth by both direct antitumor activity and anti-angiogenic activity. A pivotal phase III study reported that the response rate of imatinib-failure GIST to sunitinib was nearly $10 \%$, and the clinical benefit rate was approximately 65\%. The median PFS was 6 months, which was more than four times longer than that of the placebo arm [64]. Sunitinib is approved for the treatment of advanced GIST after failure of first-line imatinib. With regard to dosing schedule, a daily dose of $50 \mathrm{mg}$ for 4 weeks and resting for 2 weeks was initially approved. However, some patients experienced aggravation of symptoms during the resting period. A randomized, phase 2 trial was conducted to compare morning versus evening continuous dosing schedule with a daily dose of $37.5 \mathrm{mg}$ per day in imatinibfailure GIST. Combined analysis showed continuous daily sunitinib could achieve comparable efficacy as those with intermittent full dosing schedule in the literature [65]. A Chinese retrospective study also reported that the patients who received $37.5 \mathrm{mg}$ of sunitinib daily experienced less toxicity compared with $50 \mathrm{mg}$ of sunitinib scheduled 4 weeks-on and 2 weeks-off [66]. Although no randomized trials to date have compared intermittent and continuous sunitinib dosing schedules, both are equally recommended. In addition, there is no consensus on the TKI sequence (higher dose of imatinib or sunitinib) and either sequence is possible. Recent reports documented that sunitinib is associated with cardiotoxicity and hypothyroidism, therefore, close monitoring of hypertension, cardiac function, and thyroid hormones is indicated during sunitinib therapy $[67,68]$.

\section{3) Third-line regorafenib}

Regorafenib, a multikinase inhibitor of KIT, PDGFR, and VEGFR, is recommended after failure of both high-dose imatinib and sunitinib, with a regimen of $160 \mathrm{mg}$ daily for the first 3 weeks of each 4-week cycle. A randomized phase III trial (the GRID study) reported a response rate of $4.5 \%$, clinical benefit rate of nearly $50 \%$, and median PFS of 5 months with the use of regorafenib [69].

\section{4) Imatinib rechallenge}

When regorafenib is not available or failed, an imatinib rechallenge at $400 \mathrm{mg}$ per day is also recommended instead of discontinuing TKI treatment, because a certain fraction of the tumor cells may remain responsive to imatinib and disease progression can be at least slowed. With several retrospective studies suggesting a survival benefit, recent RIGHT study clearly demonstrated the benefit of imatinib rechallenge in prolongation of PFS compared to placebo after failure of at least first-line imatinib and second-line sunitinib therapies [70,71]. No conventional cytotoxic chemotherapy has ever been reported to be effective in GIST and clinical trials are under way for newer targeted agents. Thus, we do not recommend the use of these drugs, except in clinical studies [27]. 


\section{Monitoring of the imatinib plasma concentration}

Monitoring of the imatinib plasma level can be helpful for making treatment decisions in patients considered to have either dose modification or discontinuation for suboptimal response to treatment, treatment failure, unexpected serious adverse events, or suspected poor compliance. Regarding the association between imatinib plasma level and tumor response, some retrospective data from Western patients with advanced GIST have indicated that imatinib plasma levels are associated with PFS [72], while conflicting results have been reported in other prospective Western studies and retrospective Asian studies $[63,73,74]$. Therefore, the application of imatinib plasma level monitoring should currently be more focused on adjusting the dose of imatinib in the management of patients who experience intolerable toxicities due to overexposure to the drug [75]. On the other hand, prospective study for further evaluating the association between the plasma level of imatinib and clinical outcomes in GIST patients is warranted.

In this section, some points were more emphasized compared to the Western guidelines. First, since there is no consensus on the duration of adjuvant imatinib treatment after pre-operative imatinib and subsequent surgical resection, we recommend the total duration of imatinib therapy before/ after surgery to be at least 3 years for high risk GIST, while at least 2 years of post-operative imatinib is suggested in the NCCN guideline. Second, in neoadjuvant setting, we recommend early evaluation of tumor response (i.e., after the first month of imatinib treatment) when genotyping is not feasible, especially for primary gastric GIST. Third, higher dose of imatinib may be beneficial in GIST patients with KIT exon 9 mutation as recommended in the Western guidelines, but prospective study to prove the feasibility and efficacy of high dose imatinib therapy in Asian patients is needed. Lastly, if third-line regorafenib is not available or failed, imatinib rechallenge is recommended instead of discontinuing TKI treatment. However, rechallenge or continuous treatment with TKIs to which the patient has already been exposed is limited in many Asian countries unlike Western countries.

\section{Conclusion}

The paradigm of diagnosis and management of GIST has rapidly changed with the improved understanding of the pathophysiology of the tumor and the introduction of TKIs. In the late decade, several national guidelines have been published in Asian countries based on country-specific clinical situations [4-7], but there has been a strong need for a con- sensus on diagnosis and management of GISTs in Asian patients. For this reason, this first version of Asian GIST consensus guidelines was prepared through a series of meetings involving multidisciplinary experts in four countries. We hope that these guidelines will be of assistance to clinicians in Asian hospitals to improve the diagnosis and management of GIST patients and subsequently promote optimal care for this disease in Asia.

\section{Conflicts of Interest}

M.-H. Ryu is a consultant for Novartis and Bayer. A. Sawaki have honoraria from Novartis, Pfizer and Bayer, and research support from Chugai. C.-Y. Tzen received honoraria from Novartis, Pfizer and Bayer. T. Nishida received research support from Novartis and honoraria from Novartis, Pfizer, Bayer, Taiho, and Eizai. L.-T. Chen have honoraria from Novartis and Bayer, and research medication and fund from Novartis. Y.-K. Kang is a consultant for Novartis and Bayer. All other authors declare no competing interests.

\section{Author Details}

${ }^{1}$ Department of Internal Medicine, Kangbuk Samsung Hospital, Sungkyunkwan University School of Medicine, Seoul, ${ }^{2}$ Korean GIST Study Group, ${ }^{3}$ Department of Oncology, Asan Medical Center, University of Ulsan College of Medicine, Seoul, ${ }^{4}$ Department of Pathology, Samsung Medical Center, Sungkyunkwan University School of Medicine, Seoul, ${ }^{5}$ Department of Surgery, Seoul National University Hospital, Seoul National University College of Medicine, Seoul, Korea, ${ }^{6}$ Department of Gastroenterology, Japanese Red Cross

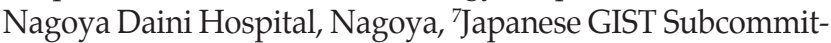
tee, ${ }^{8}$ Department of Pathology, Hyogo College of Medicine, Nishinomiya, Japan, ${ }^{9}$ Department of Pathology, Peking University Third Hospital, Beijing, ${ }^{10}$ Chinese Expert Committee on GIST, ${ }^{11}$ Department of Gastrointestinal Surgery, West China Hospital, Sichuan University, Sichuan, China, ${ }^{12}$ Department of Pathology and Laboratory Medicine, Cathay General Hospital, Taipei, ${ }^{13}$ Department of Surgery, Chang Gung Memorial Hospital and University, Taoyuan, Taiwan, ${ }^{14}$ Department of Surgery, National Cancer Center Hospital East, Chiba, Japan, ${ }^{15}$ Department of Gastrointestinal Oncology, Peking University Cancer Hospital \& Institute, Beijing, China, ${ }^{16}$ National Institute of Cancer Research, National Health Research Institutes, ${ }^{17}$ Department of Internal Medicine, National Cheng Kung University Hospital, National Cheng Kung University, Tainan, Taiwan 


\section{References}

1. Corless CL, Barnett CM, Heinrich MC. Gastrointestinal stromal tumours: origin and molecular oncology. Nat Rev Cancer. 2011;11:865-78.

2. National Comprehensive Cancer Network. NCCN clinical practice guidelines in oncology: soft tissue sarcoma, version 1 [Internet]. Fort Washington, PA: National Comprehensive Cancer Network; 2015 [cited 2015 Jun 15]. Available from: http://www.nccn.org/professionals/physician_gls/pdf/sarcoma.pdf.

3. ESMO/European Sarcoma Network Working Group. Gastrointestinal stromal tumours: ESMO Clinical Practice Guidelines for diagnosis, treatment and follow-up. Ann Oncol. 2014;25 Suppl 3:iii21-6.

4. Kang YK, Kang HJ, Kim KM, Sohn T, Choi D, Ryu MH, et al. Clinical practice guideline for accurate diagnosis and effective treatment of gastrointestinal stromal tumor in Korea. Cancer Res Treat. 2012;44:85-96.

5. Nishida T, Hirota S, Yanagisawa A, Sugino Y, Minami M, Yamamura $Y$, et al. Clinical practice guidelines for gastrointestinal stromal tumor (GIST) in Japan: English version. Int J Clin Oncol. 2008;13:416-30.

6. Expert Committee on Gastrointestinal Stromal Tumors, CSCO. China consensus on diagnosis and treatment of gastrointestinal stromal tumors (2013). Chin Clin Oncol. 2013;18:1025-34.

7. Yeh CN, Hwang TL, Huang CS, Lee PH, Wu CW, Chen-Guo $\mathrm{K}$, et al. Clinical practice guidelines for patients with gastrointestinal stromal tumor in Taiwan. World J Surg Oncol. 2012; 10:246.

8. Kim KM, Kang DW, Moon WS, Park JB, Park CK, Sohn JH, et al. Gastrointestinal stromal tumors in Koreans: it's incidence and the clinical, pathologic and immunohistochemical findings. J Korean Med Sci. 2005;20:977-84.

9. Demetri GD, von Mehren M, Antonescu CR, DeMatteo RP, Ganjoo KN, Maki RG, et al. NCCN Task Force report: update on the management of patients with gastrointestinal stromal tumors. J Natl Compr Canc Netw. 2010;8 Suppl 2:S1-41.

10. Heinrich MC, Corless CL, Demetri GD, Blanke CD, von Mehren M, Joensuu H, et al. Kinase mutations and imatinib response in patients with metastatic gastrointestinal stromal tumor. J Clin Oncol. 2003;21:4342-9.

11. Fletcher CD, Berman JJ, Corless C, Gorstein F, Lasota J, Longley BJ, et al. Diagnosis of gastrointestinal stromal tumors: a consensus approach. Hum Pathol. 2002;33:459-65.

12. Liegl B, Hornick JL, Corless CL, Fletcher CD. Monoclonal antibody DOG1.1 shows higher sensitivity than KIT in the diagnosis of gastrointestinal stromal tumors, including unusual subtypes. Am J Surg Pathol. 2009;33:437-46.

13. Kang GH, Srivastava A, Kim YE, Park HJ, Park CK, Sohn TS, et al. DOG1 and PKC-theta are useful in the diagnosis of KITnegative gastrointestinal stromal tumors. Mod Pathol. 2011;24: 866-75.

14. Kim KM, Kang DW, Moon WS, Park JB, Park CK, Sohn JH, et al. PKCtheta expression in gastrointestinal stromal tumor. Mod Pathol. 2006;19:1480-6.
15. Debiec-Rychter M, Sciot R, Le Cesne A, Schlemmer M, Hohenberger $\mathrm{P}$, van Oosterom AT, et al. KIT mutations and dose selection for imatinib in patients with advanced gastrointestinal stromal tumours. Eur J Cancer. 2006;42:1093-103.

16. Gao J, Dang Y, Sun N, Li J, Shen L. C-KIT mutations were closely associated with the response to Imatinib in Chinese advanced gastrointestinal stromal tumor patients. Med Oncol. 2012;29:3039-45.

17. Kang HJ, Ryu MH, Kim KM, Park YS, Choi J, Ryoo BY, et al. Imatinib efficacy by tumor genotype in Korean patients with advanced gastrointestinal stromal tumors (GIST): The Korean GIST Study Group (KGSG) study. Acta Oncol. 2012;51:528-36.

18. Miranda C, Nucifora M, Molinari F, Conca E, Anania MC, Bordoni $\mathrm{A}$, et al. KRAS and BRAF mutations predict primary resistance to imatinib in gastrointestinal stromal tumors. Clin Cancer Res. 2012;18:1769-76.

19. Oudijk L, Gaal J, Korpershoek E, van Nederveen FH, Kelly L, Schiavon G, et al. SDHA mutations in adult and pediatric wild-type gastrointestinal stromal tumors. Mod Pathol. 2013; 26:456-63.

20. Janeway KA, Kim SY, Lodish M, Nose V, Rustin P, Gaal J, et al. Defects in succinate dehydrogenase in gastrointestinal stromal tumors lacking KIT and PDGFRA mutations. Proc Natl Acad Sci U S A. 2011;108:314-8.

21. Miettinen M, Wang ZF, Sarlomo-Rikala M, Osuch C, Rutkowski P, Lasota J. Succinate dehydrogenase-deficient GISTs: a clinicopathologic, immunohistochemical, and molecular genetic study of 66 gastric GISTs with predilection to young age. Am J Surg Pathol. 2011;35:1712-21.

22. Miettinen M, Lasota J. Gastrointestinal stromal tumors: pathology and prognosis at different sites. Semin Diagn Pathol. 2006;23:70-83.

23. Joensuu H. Risk stratification of patients diagnosed with gastrointestinal stromal tumor. Hum Pathol. 2008;39:1411-9.

24. Rutkowski P, Bylina E, Wozniak A, Nowecki ZI, Osuch C, Matlok $\mathrm{M}$, et al. Validation of the Joensuu risk criteria for primary resectable gastrointestinal stromal tumour: the impact of tumour rupture on patient outcomes. Eur J Surg Oncol. 2011;37:890-6.

25. Joensuu H, Vehtari A, Riihimaki J, Nishida T, Steigen SE, Brabec $\mathrm{P}$, et al. Risk of recurrence of gastrointestinal stromal tumour after surgery: an analysis of pooled population-based cohorts. Lancet Oncol. 2012;13:265-74.

26. Yanagimoto $Y$, Takahashi T, Muguruma K, Toyokawa T, Kusanagi H, Omori T, et al. Re-appraisal of risk classifications for primary gastrointestinal stromal tumors (GISTs) after complete resection: indications for adjuvant therapy. Gastric Cancer. 2015;18:426-33.

27. Blay JY, Bonvalot S, Casali P, Choi H, Debiec-Richter M, Dei Tos AP, et al. Consensus meeting for the management of gastrointestinal stromal tumors: report of the GIST Consensus Conference of 20-21 March 2004, under the auspices of ESMO. Ann Oncol. 2005;16:566-78.

28. Ryu MH, Lee JL, Chang HM, Kim TW, Kang HJ, Sohn HJ, et 
al. Patterns of progression in gastrointestinal stromal tumor treated with imatinib mesylate. Jpn J Clin Oncol. 2006;36:1724.

29. Gold JS, Dematteo RP. Combined surgical and molecular therapy: the gastrointestinal stromal tumor model. Ann Surg. 2006;244:176-84.

30. Dumonceau JM, Polkowski M, Larghi A, Vilmann P, Giovannini M, Frossard JL, et al. Indications, results, and clinical impact of endoscopic ultrasound (EUS)-guided sampling in gastroenterology: European Society of Gastrointestinal Endoscopy (ESGE) Clinical Guideline. Endoscopy. 2011;43: 897-912.

31. Iwahashi M, Takifuji K, Ojima T, Nakamura M, Nakamori M, Nakatani $Y$, et al. Surgical management of small gastrointestinal stromal tumors of the stomach. World J Surg. 2006;30:2835.

32. McCarter MD, Antonescu CR, Ballman KV, Maki RG, Pisters PW, Demetri GD, et al. Microscopically positive margins for primary gastrointestinal stromal tumors: analysis of risk factors and tumor recurrence. J Am Coll Surg. 2012;215:53-9.

33. Novitsky YW, Kercher KW, Sing RF, Heniford BT. Long-term outcomes of laparoscopic resection of gastric gastrointestinal stromal tumors. Ann Surg. 2006;243:738-45.

34. Koh YX, Chok AY, Zheng HL, Tan CS, Chow PK, Wong WK, et al. A systematic review and meta-analysis comparing laparoscopic versus open gastric resections for gastrointestinal stromal tumors of the stomach. Ann Surg Oncol. 2013;20:354960.

35. DeMatteo RP, Lewis JJ, Leung D, Mudan SS, Woodruff JM, Brennan MF. Two hundred gastrointestinal stromal tumors: recurrence patterns and prognostic factors for survival. Ann Surg. 2000;231:51-8.

36. Joensuu H, Reichardt P, Eriksson M, Sundby Hall K, Vehtari A. Gastrointestinal stromal tumor: a method for optimizing the timing of CT scans in the follow-up of cancer patients. Radiology. 2014;271:96-103.

37. Desai J, Shankar S, Heinrich MC, Fletcher JA, Fletcher CD, Manola J, et al. Clonal evolution of resistance to imatinib in patients with metastatic gastrointestinal stromal tumors. Clin Cancer Res. 2007;13(18 Pt 1):5398-405.

38. Raut CP, Posner M, Desai J, Morgan JA, George S, Zahrieh D, et al. Surgical management of advanced gastrointestinal stromal tumors after treatment with targeted systemic therapy using kinase inhibitors. J Clin Oncol. 2006;24:2325-31.

39. Sym SJ, Ryu MH, Lee JL, Chang HM, Kim TW, Kim HC, et al. Surgical intervention following imatinib treatment in patients with advanced gastrointestinal stromal tumors (GISTs). J Surg Oncol. 2008;98:27-33.

40. Du CY, Zhou Y, Song C, Wang YP, Jie ZG, He YL, et al. Is there a role of surgery in patients with recurrent or metastatic gastrointestinal stromal tumours responding to imatinib: a prospective randomised trial in China. Eur J Cancer. 2014;50: 1772-8.

41. Park SJ, Ryu MH, Ryoo BY, Park YS, Sohn BS, Kim HJ, et al. The role of surgical resection following imatinib treatment in patients with recurrent or metastatic gastrointestinal stromal tumors: results of propensity score analyses. Ann Surg Oncol. 2014;21:4211-7.

42. An HJ, Ryu MH, Ryoo BY, Sohn BS, Kim KH, Oh ST, et al. The effects of surgical cytoreduction prior to imatinib therapy on the prognosis of patients with advanced GIST. Ann Surg Oncol. 2013;20:4212-8.

43. Dematteo RP, Ballman KV, Antonescu CR, Maki RG, Pisters PW, Demetri GD, et al. Adjuvant imatinib mesylate after resection of localised, primary gastrointestinal stromal tumour: a randomised, double-blind, placebo-controlled trial. Lancet. 2009;373:1097-104.

44. Li J, Gong JF, Wu AW, Shen L. Post-operative imatinib in patients with intermediate or high risk gastrointestinal stromal tumor. Eur J Surg Oncol. 2011;37:319-24.

45. Joensuu H, Eriksson M, Sundby Hall K, Hartmann JT, Pink D, Schutte J, et al. One vs three years of adjuvant imatinib for operable gastrointestinal stromal tumor: a randomized trial. JAMA. 2012;307:1265-72.

46. Agaimy A, Vassos N, Croner RS. Gastrointestinal manifestations of neurofibromatosis type 1 (Recklinghausen's disease): clinicopathological spectrum with pathogenetic considerations. Int J Clin Exp Pathol. 2012;5:852-62.

47. Bumming P, Andersson J, Meis-Kindblom JM, Klingenstierna $\mathrm{H}$, Engstrom K, Stierner U, et al. Neoadjuvant, adjuvant and palliative treatment of gastrointestinal stromal tumours (GIST) with imatinib: a centre-based study of 17 patients. Br J Cancer. 2003;89:460-4.

48. Oh JS, Lee JL, Kim MJ, Ryu MH, Chang HM, Kim TW, et al. Neoadjuvant imatinib in locally advanced gastrointestinal stromal tumors of the stomach: report of three cases. Cancer Res Treat. 2006;38:178-83.

49. Prior JO, Montemurro M, Orcurto MV, Michielin O, Luthi F, Benhattar J, et al. Early prediction of response to sunitinib after imatinib failure by $18 \mathrm{~F}$-fluorodeoxyglucose positron emission tomography in patients with gastrointestinal stromal tumor. J Clin Oncol. 2009;27:439-45.

50. Kim TW, Ryu MH, Lee H, Sym SJ, Lee JL, Chang HM, et al. Kinase mutations and efficacy of imatinib in Korean patients with advanced gastrointestinal stromal tumors. Oncologist. 2009;14:540-7.

51. Yeh CN, Chen TW, Lee HL, Liu YY, Chao TC, Hwang TL, et al. Kinase mutations and imatinib mesylate response for 64 Taiwanese with advanced GIST: preliminary experience from Chang Gung Memorial Hospital. Ann Surg Oncol. 2007;14: 1123-8.

52. Imatinib dose escalation to $800 \mathrm{mg} /$ day in Korean patients with metastatic or unresectable GIST harboring KIT exon 9 mutation (NCT01541709) [Internet]. ClinicalTrials.gov; 2015 [cited 2015 Jun 15]. Available from: http://clinicaltrials.gov/ ct2 / show / NCT01541709?term=NCT0154 1709\&rank=1.

53. Guilhot F. Indications for imatinib mesylate therapy and clinical management. Oncologist. 2004;9:271-81.

54. Blay JY, Le Cesne A, Ray-Coquard I, Bui B, Duffaud F, Delbaldo $C$, et al. Prospective multicentric randomized phase III study of imatinib in patients with advanced gastrointestinal stromal tumors comparing interruption versus continuation 
of treatment beyond 1 year: the French Sarcoma Group. J Clin Oncol. 2007;25:1107-13.

55. Lee JL, Ryu MH, Chang HM, Kim TW, Kang HJ, Sohn HJ, et al. Clinical outcome in gastrointestinal stromal tumor patients who interrupted imatinib after achieving stable disease or better response. Jpn J Clin Oncol. 2006;36:704-11.

56. Le Cesne A, Ray-Coquard I, Bui BN, Adenis A, Rios M, Bertucci $\mathrm{F}$, et al. Discontinuation of imatinib in patients with advanced gastrointestinal stromal tumours after 3 years of treatment: an open-label multicentre randomised phase 3 trial. Lancet Oncol. 2010;11:942-9.

57. Choi H, Charnsangavej C, de Castro Faria S, Tamm EP, Benjamin RS, Johnson MM, et al. CT evaluation of the response of gastrointestinal stromal tumors after imatinib mesylate treatment: a quantitative analysis correlated with FDG PET findings. AJR Am J Roentgenol. 2004;183:1619-28.

58. Choi H, Charnsangavej C, Faria SC, Macapinlac HA, Burgess MA, Patel SR, et al. Correlation of computed tomography and positron emission tomography in patients with metastatic gastrointestinal stromal tumor treated at a single institution with imatinib mesylate: proposal of new computed tomography response criteria. J Clin Oncol. 2007;25:1753-9.

59. Heinrich MC, Maki RG, Corless CL, Antonescu CR, Harlow A, Griffith D, et al. Primary and secondary kinase genotypes correlate with the biological and clinical activity of sunitinib in imatinib-resistant gastrointestinal stromal tumor. J Clin Oncol. 2008;26:5352-9.

60. Yeh CN, Chen TW, Tseng JH, Liu YY, Wang SY, Tsai CY, et al. Surgical management in metastatic gastrointestinal stromal tumor (GIST) patients after imatinib mesylate treatment. J Surg Oncol. 2010;102:599-603.

61. Zalcberg JR, Verweij J, Casali PG, Le Cesne A, Reichardt P, Blay JY, et al. Outcome of patients with advanced gastrointestinal stromal tumours crossing over to a daily imatinib dose of $800 \mathrm{mg}$ after progression on $400 \mathrm{mg}$. Eur J Cancer. 2005;41:1751-7.

62. Park I, Ryu MH, Sym SJ, Lee SS, Jang G, Kim TW, et al. Dose escalation of imatinib after failure of standard dose in Korean patients with metastatic or unresectable gastrointestinal stromal tumor. Jpn J Clin Oncol. 2009;39:105-10.

63. Yoo C, Ryu MH, Ryoo BY, Beck MY, Kang YK. Efficacy, safety, and pharmacokinetics of imatinib dose escalation to 800 $\mathrm{mg} /$ day in patients with advanced gastrointestinal stromal tumors. Invest New Drugs. 2013;31:1367-74.

64. Demetri GD, van Oosterom AT, Garrett CR, Blackstein ME, Shah MH, Verweij J, et al. Efficacy and safety of sunitinib in patients with advanced gastrointestinal stromal tumour after failure of imatinib: a randomised controlled trial. Lancet.
2006;368:1329-38.

65. George S, Blay JY, Casali PG, Le Cesne A, Stephenson P, Deprimo SE, et al. Clinical evaluation of continuous daily dosing of sunitinib malate in patients with advanced gastrointestinal stromal tumour after imatinib failure. Eur J Cancer. 2009;45: 1959-68.

66. Li J, Gao J, Hong J, Shen L. Efficacy and safety of sunitinib in Chinese patients with imatinib-resistant or -intolerant gastrointestinal stromal tumors. Future Oncol. 2012;8:617-24.

67. Chu TF, Rupnick MA, Kerkela R, Dallabrida SM, Zurakowski D, Nguyen L, et al. Cardiotoxicity associated with tyrosine kinase inhibitor sunitinib. Lancet. 2007;370:2011-9.

68. Torino F, Corsello SM, Longo R, Barnabei A, Gasparini G. Hypothyroidism related to tyrosine kinase inhibitors: an emerging toxic effect of targeted therapy. Nat Rev Clin Oncol. 2009;6:219-28.

69. Demetri GD, Reichardt P, Kang YK, Blay JY, Rutkowski P, Gelderblom H, et al. Efficacy and safety of regorafenib for advanced gastrointestinal stromal tumours after failure of imatinib and sunitinib (GRID): an international, multicentre, randomised, placebo-controlled, phase 3 trial. Lancet. 2013; 381:295-302.

70. Kang YK, Ryu MH, Yoo C, Ryoo BY, Kim HJ, Lee JJ, et al. Resumption of imatinib to control metastatic or unresectable gastrointestinal stromal tumours after failure of imatinib and sunitinib (RIGHT): a randomised, placebo-controlled, phase 3 trial. Lancet Oncol. 2013;14:1175-82.

71. Sawaki A, Kanda T, Komatsu Y, Nishida T. Impact of rechallenge with imatinib in patients with advanced gastrointestinal stromal tumor after failure of imatinib and sunitinib. Gastroenterol Res Pract. 2014;2014:342986.

72. Demetri GD, Wang Y, Wehrle E, Racine A, Nikolova Z, Blanke $\mathrm{CD}$, et al. Imatinib plasma levels are correlated with clinical benefit in patients with unresectable/metastatic gastrointestinal stromal tumors. J Clin Oncol. 2009;27:3141-7.

73. Eechoute K, Fransson MN, Reyners AK, de Jong FA, Sparreboom A, van der Graaf WT, et al. A long-term prospective population pharmacokinetic study on imatinib plasma concentrations in GIST patients. Clin Cancer Res. 2012;18:5780-7.

74. Koo DH, Ryu MH, Ryoo BY, Beck MY, Na YS, Shin JG, et al. Association of ABCG2 polymorphism with clinical efficacy of imatinib in patients with gastrointestinal stromal tumor. Cancer Chemother Pharmacol. 2015;75:173-82.

75. Yoon S, Ryu MH, Yoo C, Beck MY, Ryoo BY, Kang YK. Imatinib plasma monitoring-guided dose modification for managing imatinib-related toxicities in gastrointestinal stromal tumor patients. J Korean Med Sci. 2013;28:1248-52. 